\title{
Understanding solubility, spinnability and electrospinning behaviour of cellulose acetate using different solvent systems
}

\author{
SUSHMITA MAJUMDER ${ }^{1}$, MD ABDUL MATIN ${ }^{2}$, AHMED SHARIF $^{\mathbf{1}}$ and M TARIK ARAFAT ${ }^{3, *}$ (D) \\ ${ }^{1}$ Department of Materials and Metallurgical Engineering, Bangladesh University of Engineering and Technology (BUET), \\ Dhaka 1000, Bangladesh \\ ${ }^{2}$ Department of Glass and Ceramic Engineering, Bangladesh University of Engineering and Technology (BUET), Dhaka \\ 1000, Bangladesh \\ ${ }^{3}$ Department of Biomedical Engineering, Bangladesh University of Engineering and Technology (BUET), Dhaka 1205, \\ Bangladesh \\ *Author for correspondence (tarikarafat@bme.buet.ac.bd)
}

MS received 3 October 2018; accepted 28 January 2019; published online 23 May 2019

\begin{abstract}
The purpose of this study is to understand the solubility and spinnability of cellulose acetate (CA) and its electrospinning behaviour in different solvents. As the process of electrospinning and the corresponding fibre properties are primarily governed by the solvents used, a systematic study of the selection of solvent systems using the solubility parameters of Hildebrand and Hansen along with a Teas chart for a particular polymer is essential for the better optimization of the process. It appeared from the Teas chart that higher dispersion force $\left(f_{\mathrm{d}}\right)$ and lower hydrogen bonding force $\left(f_{\mathrm{h}}\right)$ are convenient for both the solubility and spinnability of CA in single solvent of acetone and binary solvent of 2:1 acetone/ $N, N$ dimethylacetamide (DMAc). The viscosity of the solutions escalated with increasing concentration of CA due to polymer chain entanglement which in turn favoured fibre formation. Among the solvent systems used in this work, field emission scanning electron microscopy arrayed the electrospun CA fibres using pure acetone as a solvent produced both cylindricaland ribbon-shaped fibres of a diameter of $1 \mu \mathrm{m}$, whereas CA in 2:1 acetone/DMAc yielded smooth bead-free cylindrical fibres of diameter in the range of 250-350 $\mathrm{nm}$ and CA in 3:1 acetic acid/water formed fibres with beads. Rheological analysis showed that fibre formation improved with increasing viscosity of CA solution. Electrical conductivity measurement of the CA solutions depicted that with an increase in CA concentration, fibre diameters were increased, whereas the conductivity decreased. Also, attenuated total reflectance-Fourier transform infrared spectroscopy confirmed the major peaks of CA for all the electrospun samples.
\end{abstract}

Keywords. Cellulose acetate; electrospinning; solubility; Teas chart.

\section{Introduction}

Cellulose acetate (CA) is one such polymer which brings the advantages of being abundantly available, biocompatible, biodegradable, chemically resistant and low-priced [1-5]. CA fibres fabricated by electrospinning techniques have the beneficial properties of high porosity, high surface area and roughness. By the virtue of these properties, CA electrospun fibres find their widespread applications in wound dressing, filtration, drug release, scaffolds for tissue engineering and biosensors [6-8].

While electrospinning, it has been observed that different solvent systems can yield uniform fibres without beads, defected fibres with beads, or no fibres at all [9-11]. Moreover, a good solvent for CA does not necessarily ensure its electrospinnability. Previous studies have reported that electrospinning of CA with different single and binary solvents, such as acetone, chloroform, $N, N$-dimethylacetamide (DMAc), $N, N$-dimethylformamide, acetic acid, methanol, formic acid and blends of them $[12,13]$. In these studies, variation in the molecular weight of the polymer and the nozzle size on the effect of electrospinning of CA has been analysed. Other studies extensively discussed the spinning conditions and morphologies of the obtained fibres [14-16]. However, the underlying science correlating the solubility and spinnability of CA and the necessity of binary solvents has not been addressed well so far. Hence, the present work attempted to comprehend the correlation of solubility of CA in different solvents and its electrospinnability for better controlling the electrospinning process using a Teas approach.

Teas approach has been employed in this study to interpret the solubility behaviour of CA in a single solvent system of water, acetic acid and pure acetone and binary solvent system of acetic acid/water and acetone/DMAc. To construct the Teas chart, Hildebrand and Hansen solubility parameters are used. The Hildebrand solubility parameter $(\delta)$ is the square root of the cohesive energy density (CED) of the material which indicates the relative solvency behaviour of a 
specific solvent [17-19]. Teas chart is a triangular graph which displays three categories of intermolecular forces: dispersion forces $\left(f_{\mathrm{d}}\right)$, polar forces $\left(f_{\mathrm{p}}\right)$ and hydrogen bonding forces $\left(f_{\mathrm{h}}\right)$. This chart can empirically predict the solubility behaviour of a polymer in a particular solvent based on the interaction of these intermolecular forces. Solvents, which are located close on the plot, have similar characteristics, while those located far have different solubility properties. The chart also provides an understanding of what happens in the solubility phenomena when solvents are mixed [20]. As a result, solvents, which serve the solubility of CA can be obtained from the Teas chart and the spinnability conditions of CA can be superimposed on the chart to prevent trial and error basis for selecting solvents. Recently, the Teas chart has been adopted to map the solvents for poly(vinyl alcohol) and polyimide with spinnability conditions effectively superimposed on it [21,22]. Thus, correlation of solubility and spinnability of CA by means of a Teas chart ensures reliable selection of solvents for CA. Furthermore, it would be interesting to study how the electrospinning behaviour such as, clogging during electrospinning, viscosity, electrical conductivity and fibre morphology is being affected by polymer concentration and different single and binary solvents.

In this study, the solvents of acetic acid/water, acetone/DMAc and pure acetone were primarily selected as these solvents lie at different locations of the Teas chart. This aided the study of correlating the solubility and spinnability behaviour of CA. Furthermore, different concentrations of CA were also used to investigate the effect of concentration on electrospinning behaviour and fibre morphology. Analysis of fibre morphology was performed to correlate solubility and spinnability of CA. Solution properties and process criteria such as, viscosity and electrical conductivity were also studied.

\section{Experimental}

\subsection{Materials}

CA (acetyl content $39.8 \%$ and $M_{\mathrm{n}}=30,000$ by GPC) was acquired from Sigma-Aldrich. The solvents such as acetic acid (purity 100\%), acetone (purity 100\%) and DMAc (purity 99\%) were purchased from Merck.

\subsection{Electrospinning of CA nanofibres}

CA solutions were prepared in three different solvent systems: $10,12,15,17$ and $19 \mathrm{wt} \%$ of CA in 3:1 acetic acid/water, 17 and $19 \mathrm{wt} \%$ of CA in 2:1 acetone/DMAc and 17 and $19 \mathrm{wt} \%$ of $\mathrm{CA}$ in pure acetone. A required quantity of CA was weighed and stirred with the solvents for 4-5 h at room temperature to obtain a clear solution.

The as-prepared polymer solutions were electrospun through an in-house electrospinning setup. During electrospinning, the process variables were selected based on the literature review and initial experiments [10,11]. Voltages of 17,20 and $25 \mathrm{kV}$ and feed rates of $0.5,1.5$ and $3 \mathrm{ml} \mathrm{h}^{-1}$ were employed for the CA solutions initially. A high flow rate resulted in the bead formation in spite of high voltages used. The effect of the applied voltage is influenced by three forces: coulombic force, viscoelastic force and surface tension. For moderate voltage (e.g., $20 \mathrm{kV}$ ), all the three forces remain well-balanced and result in narrow and uniform fibres, whereas the high voltage causes larger diameter fibres as solvents get less time to evaporate. From the observations and the characterizations it was quite evident that a voltage of $20 \mathrm{kV}$ and a solution flow rate of $1.5 \mathrm{ml} \mathrm{h}^{-1}$ yielded most favourable conditions for CA electrospinning. A stable jet formed without clogging the needle tip and uniform fibres without beads were collected. Similar results have also been reported elsewhere [23]. From the tip to the collector distance was fixed at $10 \mathrm{~cm}$. The electrospinning was carried out at room temperature. The collected fibre mats were dried overnight in an oven at $40^{\circ} \mathrm{C}$.

\subsection{Characterization of electrospun CA nanofibres}

The morphology of the fibres was studied using field emission scanning electron microscopy (FESEM) (JEOL JSM 7600F) at an accelerating voltage of $5 \mathrm{kV}$. Before the observations, the samples were platinum-coated using a JEOL JFC-1600 auto fine coater. The viscosity of the CA solutions was measured using a rheometer of Lamy Rheology Instruments (RM 200) with a spindle value of 2 for rotation at room temperature. The electrical conductivities of the solutions were determined with a digital electrometer (HANNA instruments, HI98312). The molecular structure and bonding nature were obtained from attenuated total reflectance-Fourier transform infrared (ATRFTIR, Cary 630, Agilent Technologies) spectra. Scanning was carried out from 650 to $4000 \mathrm{~cm}^{-1}$ with a resolution of $8 \mathrm{~cm}^{-1}$ and a scanning interval of $2 \mathrm{~cm}^{-1}$ with 32 repetitious scans averaged per sample.

\section{Results and discussion}

\subsection{Solubility and spinnability}

A systematic approach has been depicted in this work to study the solvents for CA. The solvents for CA are assessed with the Hildebrand solubility parameter, $\delta$. CA is ideally soluble in solvents or solvent systems with $\delta$ in the range of 19.43-25.57 $\mathrm{MPa}^{1 / 2}$. The values of $\delta$ for acetic acid, acetone, water and DMAc are 21.4, 19.7, 48.0 and 22.7 $\mathrm{MPa}^{1 / 2}$, respectively [20]. Hence, all the above mentioned solvents are a good choice for CA except water. The value of $\delta$ for the mixed solvent system was determined by using the following formula:

$$
\delta=\Sigma \varphi_{\mathrm{i}} \delta_{\mathrm{i}}
$$


Table 1. Hansen solubility parameters for CA and solvents [24].

\begin{tabular}{|c|c|c|c|c|c|c|}
\hline \multirow[b]{2}{*}{ Solvents } & \multicolumn{6}{|c|}{ Solubility parameter $\left(\mathrm{MPa}^{1 / 2}\right)$} \\
\hline & $\begin{array}{l}\text { Hansen } \\
\text { parameter, } \delta_{\mathrm{t}}\end{array}$ & $\begin{array}{l}\text { Dispersion } \\
\text { component, } \delta_{\mathrm{d}}\end{array}$ & $\begin{array}{l}\text { Polar } \\
\text { component, } \delta_{\mathrm{p}}\end{array}$ & $\begin{array}{l}\text { Hydrogen bonding } \\
\text { component, } \delta_{\mathrm{h}}\end{array}$ & $\begin{array}{l}\text { Polymer solubility } \\
\text { sphere, } D_{(\mathrm{s}-\mathrm{p})}\end{array}$ & $\begin{array}{l}\text { Interaction } \\
\text { radius, } R\end{array}$ \\
\hline Water & 47.83 & 15.60 & 16 & 42.3 & 32.47 & - \\
\hline Acetic acid & 21.36 & 14.50 & 8 & 13.5 & 2.68 & - \\
\hline Acetone & 19.93 & 15.50 & 10.40 & 7 & 5.39 & - \\
\hline DMAc & 22.77 & 16.80 & 11.50 & 10.20 & 5.88 & - \\
\hline 2:1 acetone/DMAc & 20.87 & 15.93 & 10.77 & 8.07 & - & - \\
\hline $3: 1$ acetic $\mathrm{acid} / \mathrm{H}_{2} \mathrm{O}$ & 27.98 & 14.78 & 10 & 20.7 & - & - \\
\hline $\mathrm{CA}$ & 19.89 & 14.90 & 7.10 & 11.10 & - & 12.40 \\
\hline
\end{tabular}

Here, $\varphi_{\mathrm{i}}$ represents the volume fraction of the solvent and $\delta_{\mathrm{i}}$ is the Hildebrand parameter of that solvent. Using this formula, $\delta$ values for 3:1 acetic acid/water and 2:1 acetone/DMAc solvent systems were found to be 28.05 and 20.70 $\mathrm{MPa}^{1 / 2}$, respectively.

Moreover, the Hansen solubility parameter has also been taken into consideration before solvent selection. The Hansen parameter $\left(\delta_{\mathrm{t}}\right)$ extended the concept of $\delta$ and divided it into three components: dispersion component $\left(\delta_{\mathrm{d}}\right)$, polar component $\left(\delta_{\mathrm{p}}\right)$ and hydrogen bonding component $\left(\delta_{\mathrm{h}}\right)$. This is expressed as follows:

$$
\delta_{\mathrm{t}}=\sqrt{ }\left(\delta_{\mathrm{d}}^{2}+\delta_{\mathrm{p}}^{2}+\delta_{\mathrm{h}}^{2}\right)
$$

Materials with adjacent values of the Hansen parameter $\left(\delta_{\mathrm{t}}\right)$ are miscible with each other. Thus, table 1 depicts that the Hansen parameter $\left(\delta_{t}\right)$ of water is $47.83 \mathrm{MPa}^{1 / 2}$ which lies far from the solubility range of CA. Also, as illustrated by Hansen, an interaction radius $(R)$ of a sphere is formed in a three-dimensional coordinate system of dispersion component $\left(\delta_{\mathrm{d}}\right)$, polar component $\left(\delta_{\mathrm{p}}\right)$ and hydrogen bonding component $\left(\delta_{\mathrm{h}}\right)$ [17]. A polymer is potentially soluble in a solvent whose interaction radius $(R)$ is greater than the distance between the solvent and the centre of the polymer solubility sphere $\left(D_{(\mathrm{s}-\mathrm{p})}\right)[20,21]$. As can be derived from table 1 , water again did not meet the criteria to be a solvent for $\mathrm{CA}$ as the interaction radius $(R)$ of $\mathrm{CA}$ is smaller than the $D_{(\mathrm{s}-\mathrm{p})}$ of water. Thus, it was evident that water has no possibility to be a solvent for CA under normal conditions [24-26].

However, CA being soluble in a solvent does not imply to its certain spinnability. Though, CA formed homogeneous solutions in all the solvents, the electrospinning of fibre was not possible with all the solutions. The Hansen method was developed by Teas where he employed a triangular chart called Teas chart or ternary solubility diagram, with CEDs to represent solubility limits. In this study, the Teas chart also served a role in understanding the electrospinnability of CA solution in terms of the fractionalparameters [27]. Teas used fractional parameters as:

$$
\begin{gathered}
f_{\mathrm{d}}=100 \delta_{\mathrm{d}} /\left(\delta_{\mathrm{d}}+\delta_{\mathrm{p}}+\delta_{\mathrm{h}}\right), \\
f_{\mathrm{p}}=100 \delta_{\mathrm{p}} /\left(\delta_{\mathrm{d}}+\delta_{\mathrm{p}}+\delta_{\mathrm{h}}\right), \\
f_{\mathrm{h}}=100 \delta_{\mathrm{h}} /\left(\delta_{\mathrm{d}}+\delta_{\mathrm{p}}+\delta_{\mathrm{h}}\right)
\end{gathered}
$$

Solubility behaviour of polymers in solvents and polymer-solvent interaction can be explicitly studied by using the solubility parameters. Previous studies reported that CA was soluble in both acetic acid and DMAc, but none of these solvents alone yielded fibres by electrospinning. Only the beaded morphology was obtained [12,28]. Thus, employing the Teas approach as shown in figure 1 , it can be deduced that DMAc and acetic acid can be used as solvents for CA as these lie closer to CA. However, from the experiment, it appeared that their solutions cannot be electrospun into fibres. As the solubility parameters of DMAc and acetic acid do not vary largely, it can be concluded that no solvent mixtures located on the straight line connecting acetic acid and DMAc are suitable for CA electrospinning. Again, as the value of dispersion force $\left(f_{\mathrm{d}}\right)$ approached acetone from DMAc, spinnability is improved. Acetone and DMAc have the solvent properties of two extremities which caused the viscosity and surface tension to lie in a moderate range after the mixing of the two. Different ratios of acetone/DMAc had been tried previously from which the ratio of 2:1 acetone/DMAc was proved to be the most versatile as it gives bead-free, continuous fibres of $\mathrm{CA}$ in the concentration range of $12.5-17 \mathrm{wt} \%$, whereas $1: 2$ acetone/DMAc showed beads [16,29].

Moreover, water cannot be used as a solvent for CA and this is evident from the Teas chart as it is located at a greater distance from CA. When $75 \%$ acetic acid was added to water, CA dissolved and could be electrospun leading to beaded fibres. It is apparent from the chart that the binary system of acetic acid and water containing $<75 \%$ acetic acid will be inappropriate for electrospinning of CA as it will lead to an overall lower dispersion force, $f_{\mathrm{d}}$. For binary solvents, if the point on the line connecting the two constituent single solvents lie close to that solvent suitable 


\section{Solubility and Spinnabilty of $17 \%$ CA}

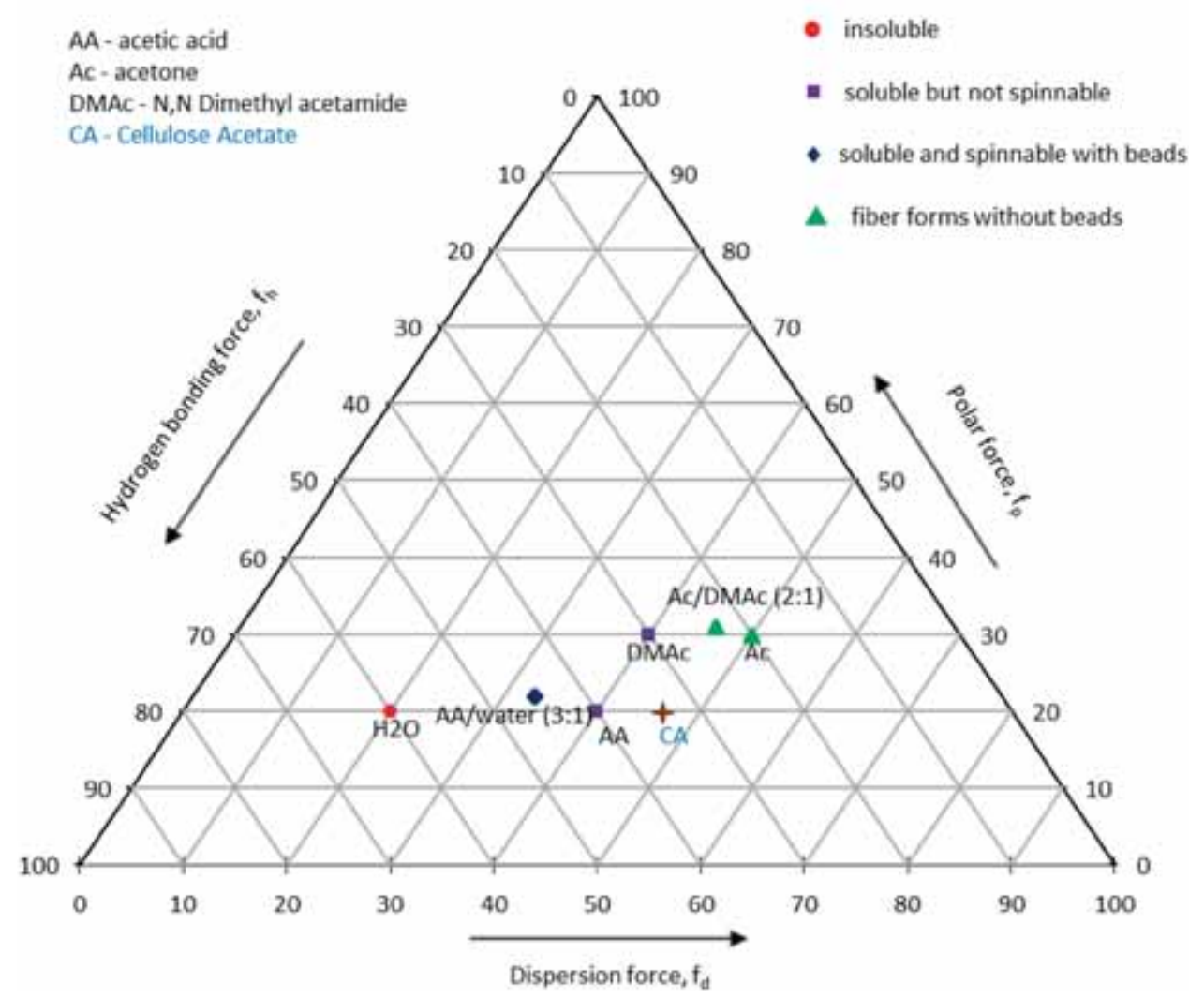

Figure 1. Teas chart to analyse the feasibility of solution formation and spinning with different solvents for CA.

for electrospinning can be regarded as a suitable binary solvent system for the process. Thus, the importance of binary solvent systems becomes evident as two solvents which were previously incompatible for spinning, after blending, developed a solvent system convenient for electrospinning. Hence, the solvents with the lower value of dispersion force $\left(f_{\mathrm{d}}\right)$ and a higher value of hydrogen bonding force $\left(f_{\mathrm{h}}\right)$ and polar force $\left(f_{\mathrm{p}}\right)$ are not suitable for solution forming and spinning of CA. When a mixture of acetic acid/water was explored, fibre formed with beads as water caused dissociation of acetic acid necessary for spinning. In this case, hydrogen bonding force $\left(f_{\mathrm{h}}\right)$ did not show any significant effect. It can be interpreted that the interaction of dispersion force $\left(f_{\mathrm{d}}\right)$, hydrogen bonding force $\left(f_{\mathrm{h}}\right)$ and polar force $\left(f_{\mathrm{p}}\right)$ played a vital role in determining spinnability. Thus, the Teas approach can be used as an effective guideline to differentiate between solvents for electrospinning and non-solvents for CA.

\subsection{Viscoelastic properties of CA solution}

The solution properties of CA dictate the electrospinning behaviour. A minimum concentration of the polymer is required to cause polymer chain entanglement. Below this minimum concentration, polymer chains overlap, but no entanglement takes place which is crucial for electrospinning.
It has been seen for CA that increasing the concentration favoured fibre formation. This change in concentration is associated with the rheological behaviour of the solution which controls the morphology of the electrospun fibre. Viscosity is considered to be the key parameter which decides the fibre diameter and whether electrospinning will result in fibre formation. The viscosities of 10, 12, 15 and $17 \mathrm{wt} \%$ $\mathrm{CA}$ in 3:1 acetic acid/water were gradually increased with increasing the CA concentration. This is due to the increased number of CA molecules and its entanglement in the solution. The increase in viscosity leads to high viscoelasticity which matches with the electrostatic and coulombic repulsive forces associated with the stretching of the electrospinning jet $[19,30]$. However, when the viscosity is too high, the control of the polymer flow to the tip of the needle is prohibited by the cohesive nature of the solution $[13,31]$. The shear stress $v s$. shear rate as shown in figure 2 also demonstrated that the shear thickening effect of CA in both the binary solvent systems.

The formation of a stable jet during electrospinning plays a vital role in the formation of fibres and also depends on the viscoelasticity of the solution. However, this jet is influenced by the process parameters which, if not controlled, can lead to jet splitting and the formation of beaded fibres [32]. Beads are considered as defects as they reduce the active surface area of fibres. 


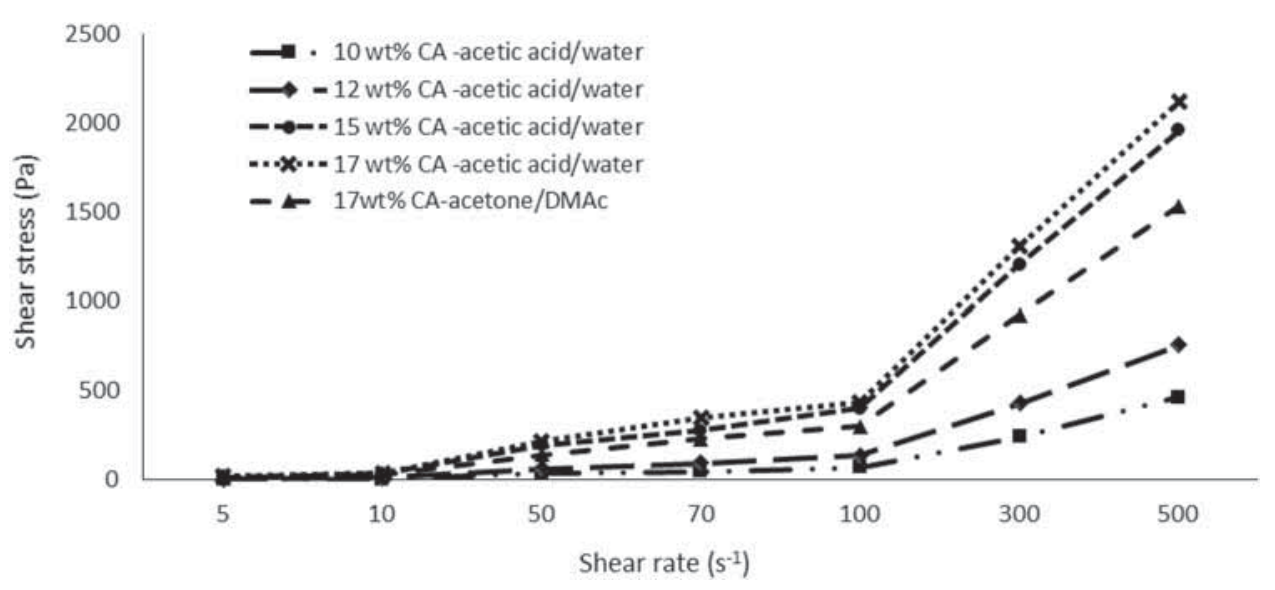

Figure 2. Relationship between the shear stress and shear rate of CA solutions with varied concentrations and solvent systems.
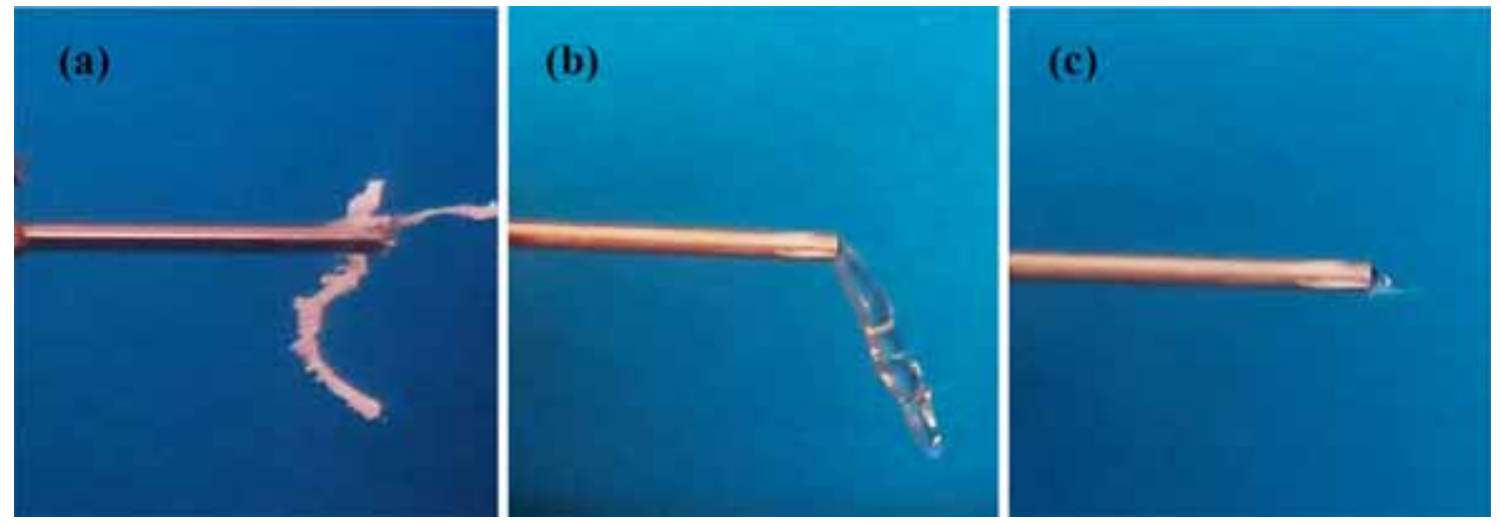

Figure 3. Different needle tip conditions showing (a) clogging of the needle tip for CA in pure acetone, (b) bending elongation of jet for 10 and $12 \mathrm{wt} \% \mathrm{CA}$ in 3:1 acetic acid/water and (c) formation of stable cone for 15, 17 and $19 \mathrm{wt} \%$ CA in binary solvents.
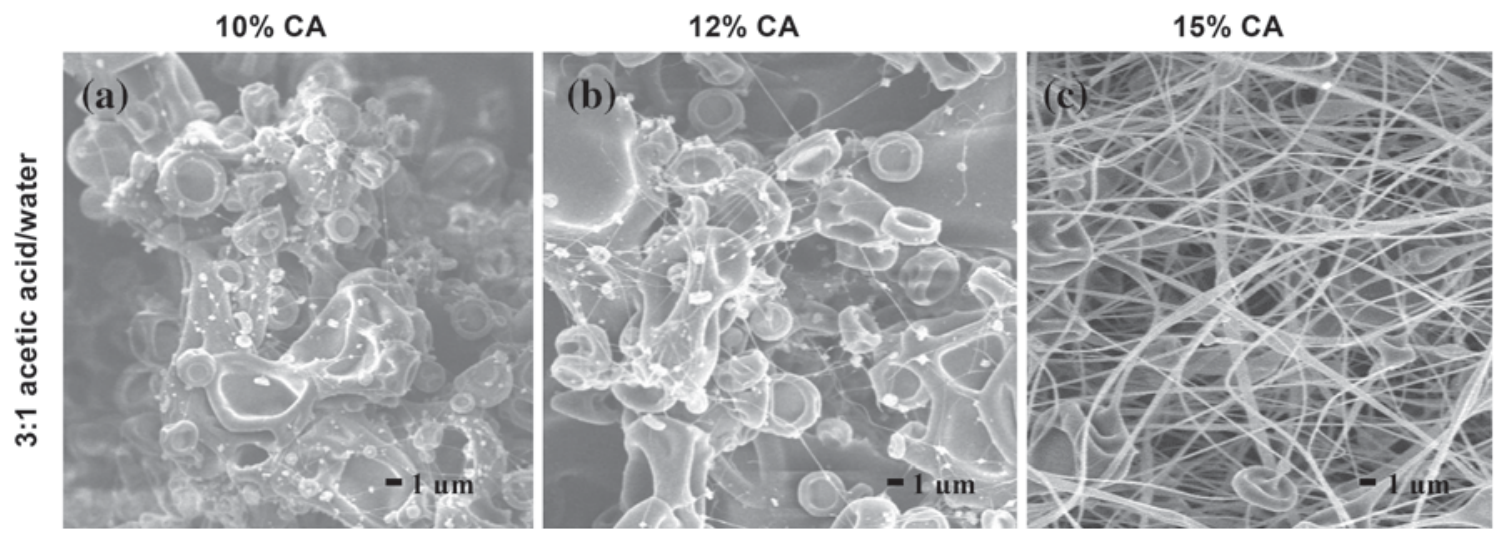

Figure 4. SEM images of (a) 10, (b) 12 and (c) $15 \mathrm{wt} \%$ CA in 3:1 acetic acid/water.

Figure $3 \mathrm{a}$ shows the bending elongated jet which was formed with the 10 and $12 \mathrm{wt} \% \mathrm{CA}$ solutions. The electrically driven bending instabilities can be explained by the Earnshaw theorem of electrostatics which states that a stable structure cannot be created where the elements of the structure connect only by Coulomb's law. The Coulomb's interaction energy is minimized by moving the polymer jet in a complicated way by the virtue of its in-built charges [33,34]. The 


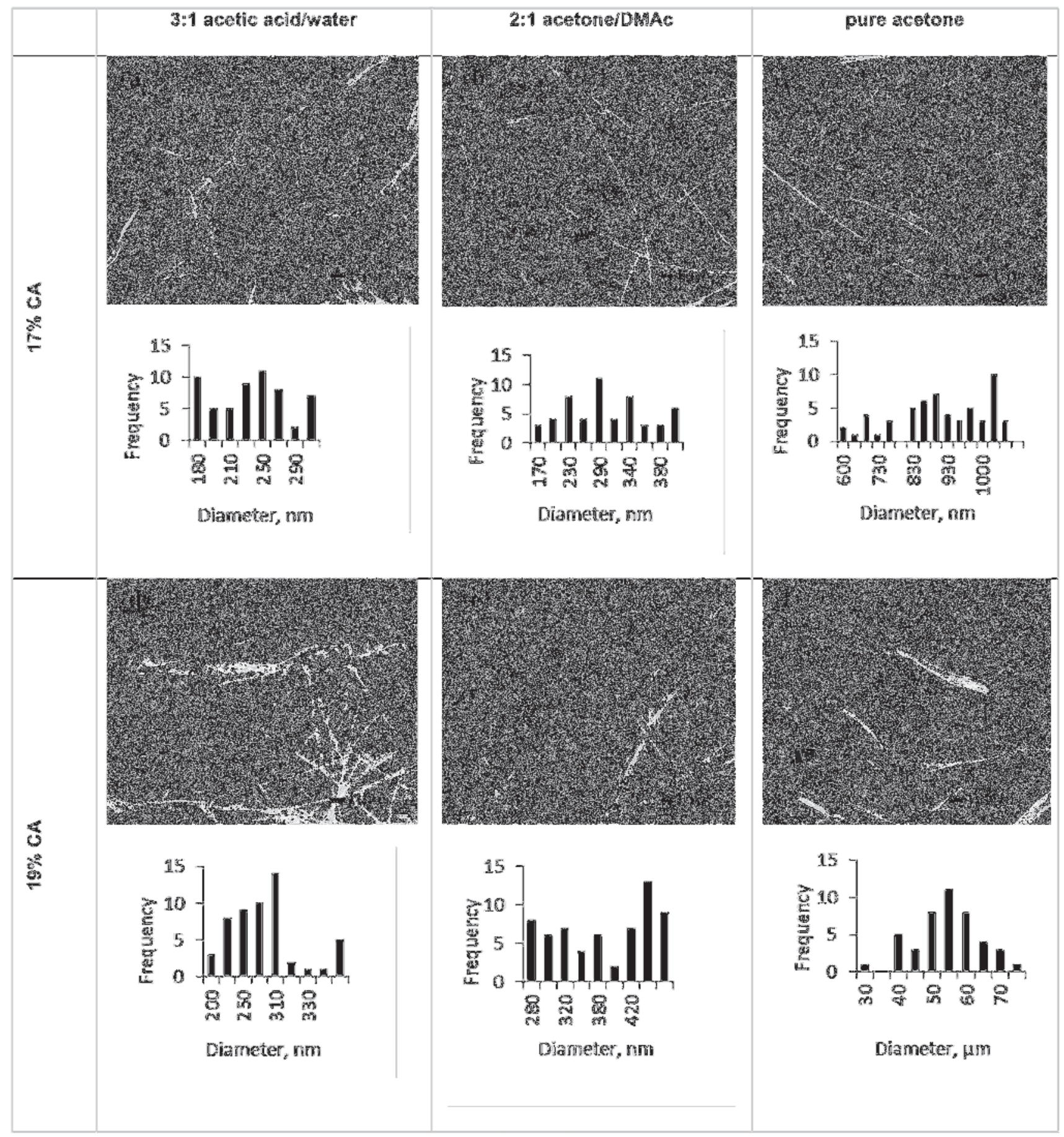

Figure 5. SEM images and fibre size distributions of 17 wt \% CA in (a) 3:1 acetic acid/water, (b) 2:1 acetone/DMAc and (c) pure acetone; and $19 \mathrm{wt} \% \mathrm{CA}$ in (d) 3:1 acetic acid/water, (e) 2:1 acetone/DMAc and (f) pure acetone.

probable reason for the bending of jet, while spinning with 10 and $12 \mathrm{wt} \% \mathrm{CA}$ can be associated with the increasing repulsive forces between the charges carried by the jet which caused every portion of it to lengthen along a changing direction until the jet solidified [9]. The pendant droplet becomes stable when there is equilibrium between the surface tension of the solution and the electric forces [35,36]. Splitting and blockade at the needle tip occurred as shown in figure $3 \mathrm{~b}$ when the process was carried out with pure acetone. Due to the high volatility of acetone, it evaporated as soon as it emerged through the needle and clogged the needle tip. This resulted in a tedious task of cleaning the needle tip at regular intervals. The stable jet formed (figure 3c) when the CA concentration increased and solvent systems included 3:1 acetic acid/water 
Table 2. Process parameters and solvent systems for electrospinning CA.

\begin{tabular}{lllc}
\hline CA concentration (wt\%) & Solvents & SEM observation & Diameter (nm) \\
\hline 10 & $3: 1$ acetic acid/water & Only beads, no fibre & - \\
12 & $3: 1$ acetic acid/water & Comparatively smaller beads with few fibres & $35-50$ \\
15 & $3: 1$ acetic acid/water & Fibre forms with small beads & 200 \\
17 & $3: 1$ acetic acid/water & More uniform fibres with fewer beads & $180-280$ \\
19 & $3: 1$ acetic acid/water & Fibres with elongated beads & $220-310$ \\
17 & $2: 1$ acetone/water & Smooth, uniform fibres, no beads & $250-350$ \\
19 & $2: 1$ acetone/water & Homogeneous, bead-free fibres & $280-450$ \\
17 & Pure acetone & Cylindrical and flat fibres, no beads & $800 \mathrm{~nm}-1.2 \mu \mathrm{m}$ \\
19 & Pure acetone & Flat fibres, no beads & $50-65 \mu \mathrm{m}$ \\
\hline
\end{tabular}

or 2:1 acetone/DMAc. This is the condition when an increase in the viscosity prevented the partial breakup of the jet and the surface tension was successfully surmounted to form fibres [4].

\subsection{Morphological study of electrospun CA fibre}

The morphological observation shown in figure $4 \mathrm{a}$ and $\mathrm{b}$ revealed that 10 and $12 \mathrm{wt} \%$ of CA in 3:1 acetic acid/water did not generate any fibres. Only large beads were observed. This is attributed to the lack of polymer chain entanglement in the solution. As the concentration of CA increased to $15 \mathrm{wt} \%$ beaded fibres begin to form as shown in figure 4c. With the increase in CA concentration, viscosity increased and this drove the polymer chain entanglement to stabilize the jet. Higher viscosity favours the formation of fibres without beads $[8,37,38]$. However, the fibre diameters did not alter much on increasing CA concentration in acetic acid/water. The previous study reported that DMAc could not produce fibres, but when mixed with acetone, fibres were obtained. Due to the presence of acetone, high viscosity and surface tension of DMAc are reduced and formed favourable conditions for fibre formation [16]. Seventeen wt $\%$ CA in 3:1 acetic acid/water formed fibres with beads, 2:1 acetone/DMAc generated cylindrical bead-free fibres and pure acetone constituted of a blend of cylindrical and flat fibres of diameter $\sim 1 \mu \mathrm{m}$ as depicted in figure $5 \mathrm{a}-\mathrm{c}$. The 19\% CA yielded bead-free fibres with 2:1 acetone/DMAc, however, failed to do so with $3: 1$ acetic acid/water (figure $5 \mathrm{~d}$ and e). Furthermore, when $19 \mathrm{wt} \% \mathrm{CA}$ in pure acetone was electrospun, a significant change in the fibre morphology has been observed. Flat fibres with a creased surface and higher transverse sections of 50-60 $\mu \mathrm{m}$ with fused junctions were obtained in this case (figure 5f). Ribbon or flat-shaped fibres are due to the fast evaporation of volatile acetone. It has been reported that the tubular fibre skin gets collapsed when a highly volatile solvent is used leading to ribbon-like fibres [24]. Therefore, it can be said that different solvent systems lead to different morphologies. Table 2 summarizes the observed morphology of the electrospun

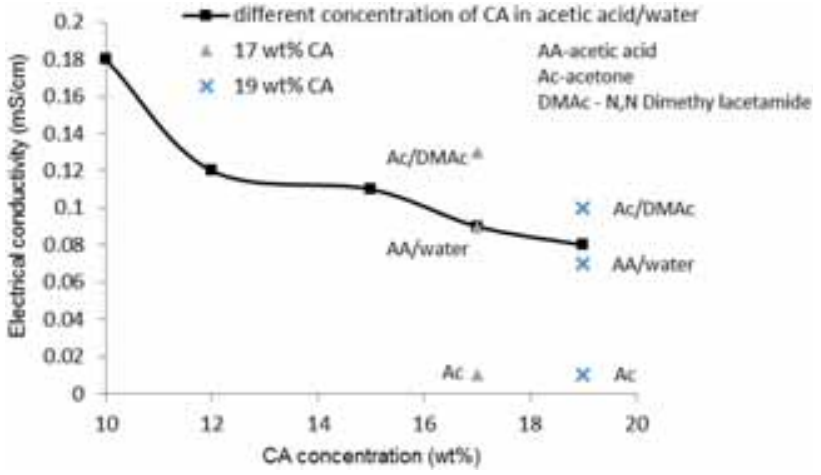

Figure 6. Electrical conductivity of CA for different concentrations and solvent systems.

samples of CA with the solvent systems and electrospinning conditions.

\subsection{Effects of electrical conductivity of solution}

A minimum electrical conductivity of the solution is required for electrospinning to take place. An electric field is created between the polymer solution and the collector which causes stretching of the polymer jet towards the grounded collector. Electrical conductivity indicates the available electrical charges on the surface of the polymer solution [37]. The electrical conductivity measured for CA showed a decrease in conductivity with an increase in CA concentration (figure 6). This was due to the decreased charge density in the solution as the amount of dissociated ions was reduced with an increase in the concentration due to the nonionic nature of CA. The higher concentration of $\mathrm{CA}$ also hindered the jet elongation and produced fibres of larger diameter. The mass deposition rate and initial jet diameter during electrospinning decrease with an increase in the surface charge density and this fact aligned with the explanation of decrease in the fibre diameter with an increase in electrical conductivity [20,39]. 


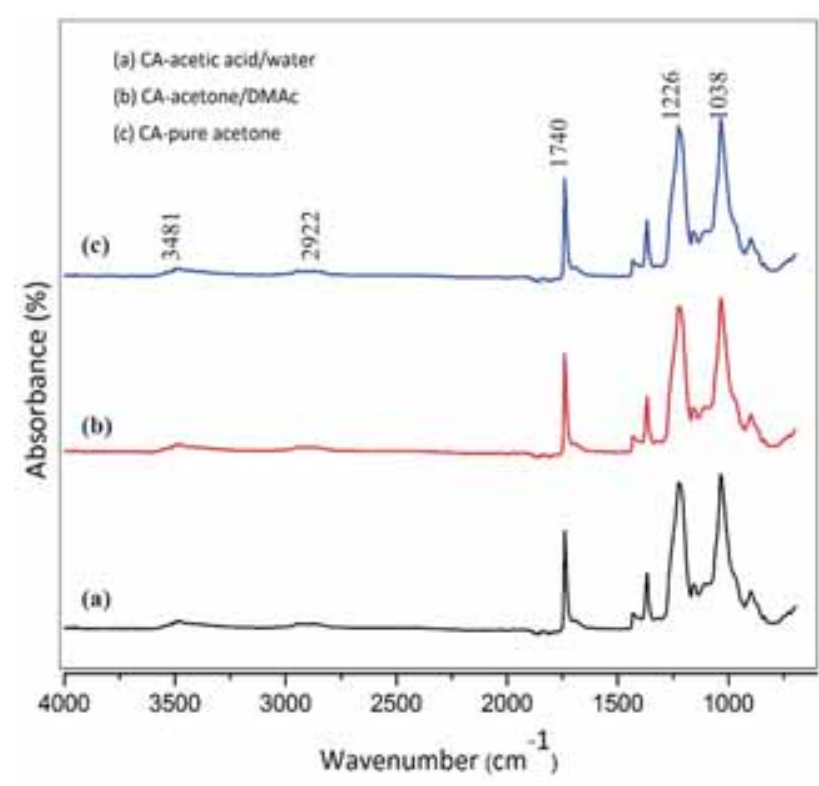

Figure 7. ATR-FTIR spectrum of CA for different solvent systems.

\section{$3.5 \quad$ ATR-FTIR}

ATR-FTIR spectra of the fibre mat spun with different solvents show the similar major peaks of CA, which explained that there were no chemical changes during the formation of solution. CA produced broad absorption bands at 3481 and $2922 \mathrm{~cm}^{-1}$ indicating $\mathrm{O}-\mathrm{H}$ groups and a sharp peak at $1740 \mathrm{~cm}^{-1}$ associated with the carbonyl band is the characteristic peak of CA as other peaks may appear in the case of cellulose as well. There were also symmetric and antisymmetric bendings of methylene groups at 1038 and 1226 $\mathrm{cm}^{-1}$, respectively, as shown in figure $7[19,25,40]$. No peaks of solvents were observed due to their evaporation during electrospinning and subsequent heating at $40^{\circ} \mathrm{C}[41,42]$.

\section{Conclusion}

A systematic approach has been illustrated in this work for the analysis and selection of solvents for electrospinning of CA. The Teas approach aided in analysing the solvent systems which paved the way for better correlating of CA solubility and spinnability in a new dimension, previously not illustrated elsewhere. CA nonwoven fibre mats were synthesized by electrospinning using 3:1 acetic acid/water, 2:1 acetone/DMAc and pure acetone as solvent systems. Electrospinning with acetone generated difficulties due to needle tip blockage, while stable jet formed with acetic acid/water and acetone/DMAc. It was realized that 10 and $12 \mathrm{wt} \% \mathrm{CA}$ did not produce any fibres except beads in the acetic acid/water system due to low viscosity and high surface tension. A minimum of $15 \mathrm{wt} \% \mathrm{CA}$ is needed to establish polymer chain entanglement to form a nonwoven fibre. Higher CA content increased the viscosity and also the fibre diameters. However, electrical conductivity is found to decrease with an increase in diameter. This is implied to the fact that the diameter increases with an increase in CA concentration which in turn reduces surface charge density and results in lower electrical conductivity. The electrospinning of CA in acetone showed quite a different morphology. It is composed of both cylindrical and ribbon-like structures with a diameter of $1 \mu \mathrm{m}$ with $17 \mathrm{wt} \%$ $\mathrm{CA}$, and a completely flat morphology with a creased fibre surface is obtained with $19 \mathrm{wt} \%$ CA. Thus, it is anticipated that this study will assist in better understanding of CA electrospinning and the consequences of solution properties on the characteristics of electrospun fibres by the methodical study of the solvents suitable for both solubility and spinnability of CA.

\section{Acknowledgements}

Financial support is greatly acknowledged from the Ministry of Science and Technology, Bangladesh under the grant no. 39.00.0000.09.06.79.2017/EAS-393.

\section{References}

[1] Konwarh R, Karak N and Misra M 2013 Biotechnol. Adv. 31 421

[2] Lee H, Nishino M, Sohn D, Lee J S and Kim I S 2018 Cellulose 252829

[3] Khoshnevisan K, Maleki H and Samadian H 2018 Carbohydr. Polym. 198131

[4] Tarus B, Fadel N, Al-Oufy A and El-Messiry M 2016 Alexandria Eng. J. 198131

[5] Otsuka I, Njinang C N and Borsali R 2017 Cellulose 243281

[6] Gouda M, Aljaafari A and Al-Omair M A 2017 Fiber Polym. 182379

[7] Teo W E and Ramakrishna S 2006 Nanotechnology 1789

[8] Agarwal S, Wendorff J H and Greiner A 2008 Polymer (Guildf) 495603

[9] Shenoy S L, Bates W D and Wnek G 2005 Polymer (Guildf) 468990

[10] Yang G, Li X, He Y, Ma J, Ni G and Zhou S 2018 Polym. Sci. 8180

[11] Pham Q P, Sharma U and Mikos A G 2006 Tissue Eng. 121197

[12] Tungprapa S, Puangparn T, Weerasombut M, Jangchud I, Fakum P, Semongkhol S et al 2007 Cellulose 14563

[13] Son W K, Youk J H, Lee T S and Park W H 2004 J. Polym. Sci. B: Polym. Phys. 425

[14] Liu H and Tang C 2006 Polym. J. 3965

[15] Celebioglu A and Uyar T 2011 Mater. Lett. 652291

[16] Zhang K, Li Z, Kang W, Deng N, Yan J, Ju J et al 2018 Carbohydr. Polym. 18362

[17] Elidrissi A, El-Barkany S, Amhamdi H, Maaroufi A and Hammouti B 2012 J. Mater. Environ. Sci. 3270

[18] Marcus Y 2018 J. Chem. Thermodyn. 126187

[19] Klar F and Urbanetz N A 2016 Drug Dev. Ind. Pharm. 421621 
[20] Luo C J, Nangrejo M and Edirisinghe M 2010 Polymer (Guildf) 511654

[21] Mahmud M M, Perveen A, Matin M A and Arafat M T 2018 Mater. Res. Express 5115407

[22] Lasprilla-Botero J, Álvarez-Láinez M and Lagaron J M 2018 Mater. Today Commun. 141

[23] Huan S, Liu G, Han G, Cheng W, Fu Z, Wu Q et al 2015 Materials 82718

[24] Ghorani B, Russell S J and Goswami P 2013 Int. J. Polym. Sci. 20131

[25] Batista M M, Guirardello R and Krähenbühl M A 2015 J. Am. Oil Chem. Soc. 9295

[26] Han S O, Youk J H, Min K D, Kang Y O and Park W H 2008 Mater. Lett. 62759

[27] Lee T L and Lo C T 2017 J. Polym. Sci. 55793

[28] Ghorani B, Goswami P and Russell S J 2015 Res. J. Text. App. 1924

[29] Brown T D, Dalton P D and Hutmacher D W 2016 Prog. Polym. Sci. 56116

[30] Haider A, Haider S and Kang I K 2018 Arab. J. Chem. 11 1165
[31] Rutledge G C and Fridrikh S V 2007 Adv. Drug Deliv. Rev. 59 1384

[32] Hohman M M, Shin M, Rutledge G C and Brenner M P 2001 Phys. Fluids 132221

[33] Fathona I W and Yabuki A 2016 Fiber Polym. 171238

[34] Wu Y, Wang L, Fan J, Shou W, Zhou B M and Liu Y 2018 Polymers 10572

[35] Hoogesteijn von Reitzenstein N, Bi X, Yang Y, Hristovski K and Westerhoff P 2016 J. Appl. Polym. Sci. 13343811

[36] Wu S, Qin X and Li M 2014 J. Ind. Text. 4485

[37] Kurtz I and Schiffman J 2018 Materials 111059

[38] Tan S H, Inai R, Kotaki M and Ramakrishna S 2005 Polymer 466128

[39] Strager J, Tucker N, Kirwan K and Staiger M P 2009 Int. J. Mod. Phys. B 231956

[40] Ma Z, Kotaki M and Ramakrishna S 2005 J. Memb. Sci. 265 115

[41] Ramakrishna S, Fujihara K, Teo W E, Yong T, Ma Z and Ramaseshan R 2006 Mater. Today 940

[42] Ao C, Niu Y, Zhang X, He X, Zhang W and Lu C 2016 Int. J. Biol. Macromol. 97568 